\title{
Implementación del algoritmo reverso para el cribado de sífilis en un laboratorio clínico
}

\author{
M. Soledad Martínez Methol', Fernando D. Ventimiglia1,2,3, Ana M. Aristimuño', \\ Amelia N. de la Colina', Jorge J. Bruno' y Liliana E. D'Agostino'
}

\section{Implementation of the reverse screening syphilis algorithm in a clinical laboratory}

Background: Syphilis remains a public health concern worldwide, the accuracy of diagnostic tests is critical for its successful control. Currently, there are two approaches to the diagnosis of syphilis using serological tests: the traditional algorithm and the reverse algorithm. Aim: The goal of this study was to analyse the advantages and disadvantages in the implementation of the syphilis reverse-screening algorithm in an outpatient clinical laboratory. Methods: An observational cross-sectional study was carried out analyzing 246 reactive sera from a total of 14700 requests for syphilis serology. Chemiluminescent assay ARCHITECT Syphilis TP, V.D.R.L. and FTA-Abs were performed. Results: Among 246 reactive sera by ARCHITECT Syphilis TP, 129 were reactive and 117 were non-reactive by V.D.R.L. the last mentioned resulted in 97 reactive and 20 non-reactive by FTA-Abs, suggesting false positives $(0.13 \%)$. Two patients with primary infection were detected, that were not detected by V.D.R.L. and one pregnant woman with primary infection with a high value S/CO and V.D.R.L.:1 dils. Conclusions: Among the advantages of using a reverse algorithm were greater sensitivity in the detection of patients with primary syphilis; automation, complete traceability of the samples; objective interpretation and conclusive results.

Keywords: Syphilis; reverse algorithm for syphilis; treponemal chemiluminescence immunoassay.

Palabras clave: Sífilis; algoritmo reverso para sífilis; inmunoensayo treponémico quimioluminiscente.

\section{Introducción}

$\mathrm{L}$ a sífilis, causada por la espiroqueta Treponema pallidum subespecie pallidum, es una enfermedad venérea de presentación variada y compleja. Puede ser transmitida por medio de unas pocas vías: contacto sexual, introducción directa en el aparato vascular por medio de agujas compartidas y transfusiones infectadas o transferencia transplacentaria de espiroquetas. La enfermedad clínica se dividió en una serie de estadios clínicos sintomáticos secuenciales, separados por períodos de infección latente: período de incubación, primario, secundario, tardío o terciario y sífilis congénita cuando la transmisión es vertical ${ }^{1}$.

Para el diagnóstico clínico es mandatorio el uso de adecuados métodos de laboratorio y la interpretación de los resultados en base a la historia clínica del paciente y el examen físico. Treponema pallidum no puede ser cultivado in vitro por lo que se han desarrollado varias técnicas diagnósticas, directas e indirectas, no existiendo aún un único ensayo para el diagnóstico ${ }^{2}$.

Los métodos directos, incluyen microscopía de campo oscuro en muestras de fluidos o frotis de lesiones, análisis con anticuerpos fluorescentes en muestras de lesiones o secciones de tejidos y reacción de polimerasa en cadena (RPC).
El diagnóstico indirecto se basa en técnicas inmunológicas para la detección de anticuerpos, no existiendo aún un método estándar de oro universalmente aceptado. Tradicionalmente se han utilizado los métodos no treponémicos en el cribado y los treponémicos como confirmatorios (Figura 1). Todos los métodos no treponémicos miden anticuerpos anti-fosfolipídicos totales formados por el hospedero en respuesta a los lípidos de las superficies celulares de los treponemas (reaginas sifilíticas) y utilizan como antígenos una mezcla de cardiolipinas, lecitina y colesterol; entre éstos encontramos los ensayos serológicos ampliamente usados, con base en la técnica de Wassermann (1906), como la reacción de reagina plasmática rápida (RPR), Laboratorio de Investigación de Enfermedades Venéreas (V.D.R.L.) y V.D.R.L. modificado o reagina sérica sin calentar (USR). Entre las técnicas inmunológicas treponémicas, que detectan anticuerpos específicos de treponema, se han empleado: absorción de anticuerpos treponémicos fluorescentes (FTA-Abs) y ensayos de microhemaglutinación y aglutinación de partículas para T. pallidum (MHA-TP; TPPA) ${ }^{3,4}$.

Durante la última década, se han desarrollado nuevos ensayos serológicos treponémicos usando antígenos específicos (nativos y recombinantes) y nuevos formatos, que incluyen métodos rápidos basados en inmunocromatografía, inmunoensayos enzimáticos
'Laboratorio D'Agostino-Bruno, La Plata, Argentina.

${ }^{2}$ Cátedra Hematología, FCE, UNLP.

${ }^{3}$ Cátedra Hematología, ICS, UNAJ

Los autores declaran que no

existieron conflictos de interés en el presente estudio.

No hubo fuentes de financiación externas.

Recibido: 8 de abril de 2018 Aceptado: 4 de junio de 2019

Correspondencia a:

María Soledad Martínez Methol smartinezmethol@dagostinobruno.com.ar 


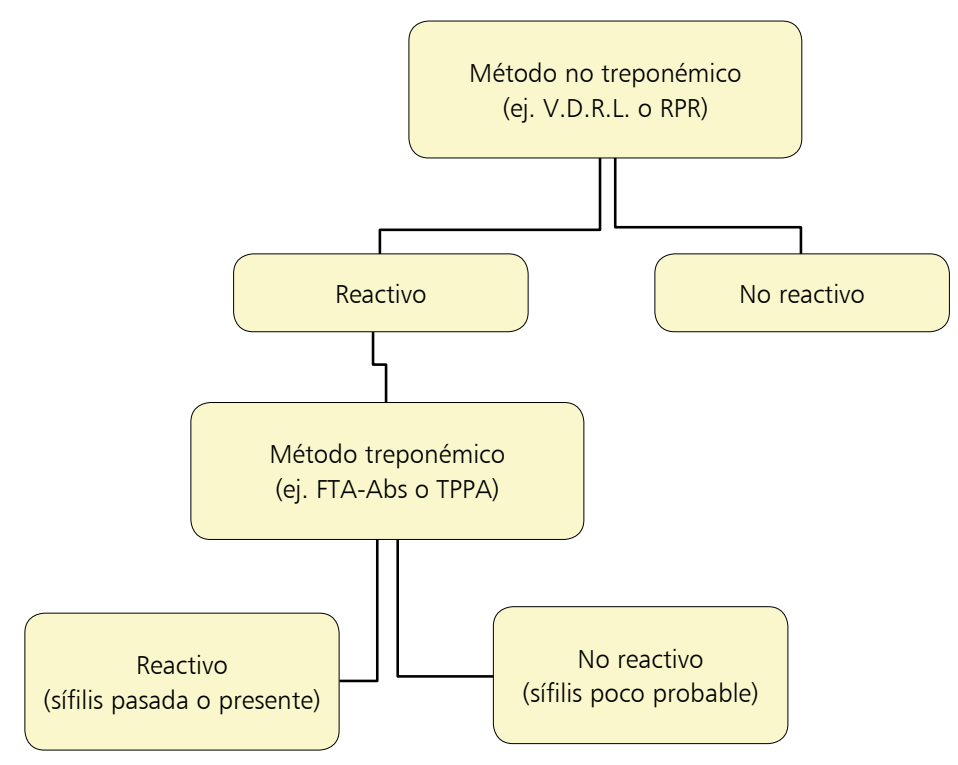

Figura 1. Algoritmo tradicional para el cribado de sífilis.

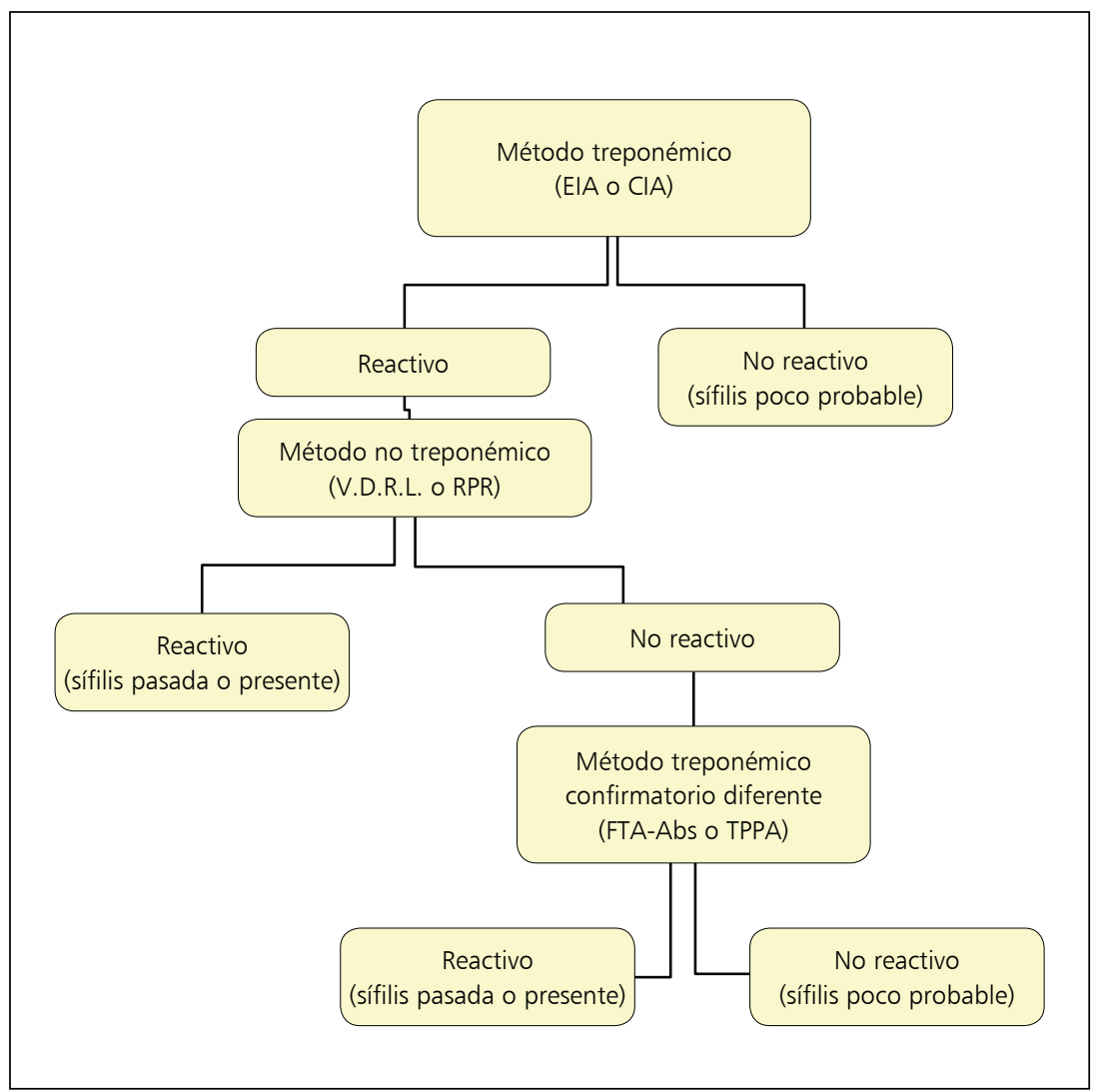

Figura 2. Algoritmo reverso para el cribado de sífilis. y quimioluminiscentes (EIA; CIA), muchos de éstos totalmente automatizados, asegurando trazabilidad de las muestras y objetividad en el resultado, constituyendo la base para establecer el algoritmo de cribado de secuencia reversa, en el cual las muestras son analizadas con un ensayo treponémico seguido de un análisis de los sueros reactivos con un ensayo no treponémico ${ }^{5}$ (Figura 2). Este algoritmo reverso constituye un cambio notable en la interpretación del diagnóstico serológico ya que la respuesta inmunológica de los métodos no treponémicos disminuye con el tratamiento hasta ser indetectables mientras que los ensayos inmunológicos treponémicos permanecen siempre positivos.

Es importante que los profesionales de la salud conozcan los fundamentos de estos métodos, su alcance, interpretación e implicancias para la salud pública ${ }^{5,6}$. La Organización Panamericana de la Salud (OPS) y el Centro para el Control y Prevención de Enfermedades (CDC) continúan recomendando el algoritmo tradicional con cribado no treponémico; sin embargo, el CDC estableció recomendaciones para los laboratorios que utilicen el algoritmo reverso ${ }^{7-9}$. El cribado con ensayos treponémicos es recomendado por el Centro para el Control y Prevención de Enfermedades Europeo (ECDC) ${ }^{10}$. El objetivo de este estudio fue analizar las ventajas y desventajas en la implementación del algoritmo reverso en un laboratorio clínico de pacientes ambulatorios.

\section{Materiales y Métodos}

Se realizó un estudio de corte transversal, observacional, analizando 246 sueros reactivos por el método quimioluminiscente de cribado sobre un total de 14.700 solicitudes de serología para sífilis, que fueron recibidas en nuestro laboratorio durante el período septiembre de 2014 hasta enero de 2017. La identidad de los pacientes no fue revelada y los datos procesados anónimamente (Tabla 1). Los requerimientos pre-analíticos para la obtención de las muestras de sangre fueron: punción venosa entre las 7:00 y 10:00 am, ocho horas de ayuno, coagulación de 30 minutos a temperatura ambiente en tubos con gel acelerador de la coagulación y centrifugación durante 15 min a $3.600 \mathrm{rpm}$. Los sueros así obtenidos, se procesaron con las muestras diarias de rutina y/o se guardaron a $4^{\circ} \mathrm{C}$ hasta su procesamiento durante un lapso de tiempo que no superó los siete días según las recomendaciones del fabricante para la estabilidad de los sueros. El cribado treponémico se realizó con el ensayo ARCHITECT Syphilis TP (Abbott, Alemania), con principio en un inmunoanálisis quimioluminiscente de micropartículas (CMIA) para la detección cualitativa de anticuerpos frente a T. pallidum empleando antígenos recombinantes (TpN15, TpN17 y TpN47). El ensayo 
compara la señal de la muestra y el punto de corte (S/ $\mathrm{CO}$ ) interpretando los valores $\mathrm{S} / \mathrm{CO} \geq 1$ como reactivos. La verificación del desempeño del método fue realizada por nuestro laboratorio en un estudio previo ${ }^{11}$. Todos los sueros reactivos $(n=246)$ en el cribado fueron posteriormente analizados con el ensayo reflejo no treponémico V.D.R.L. modificado (Wiener Lab, Argentina). Un total de 117 sueros resultaron no reactivos por el método no treponémico y fueron a continuación analizados con la técnica FTA-Abs (Biocientífica S.A., Argentina) según indica el algoritmo reverso (Figura 3).

El procedimiento de control de calidad interno para el ensayo ARCHITECT Syphilis TP se realizó según recomendaciones del fabricante, procesando una muestra única de control reactivo y no reactivo cada día de su uso y para cada lote de reactivos. En la realización de la técnica FTA-Abs se procesaron muestras reactivas y no reactivas con cada impronta usada. Cuando se utilizó el método V.D.R.L. modificado se empleó un pool de sueros reactivos de título dos diluciones y un pool de sueros no reactivos, procesados por distintos operadores. El control de calidad externo se realizó mensualmente mediante el programa ProgBA-CEMIC y trimestralmente con el Programa de Evaluación Externa de Calidad de la Fundación Bioquímica Argentina (PEEC), obteniendo resultados aceptables en el período evaluado.

Se realizó el cálculo de sensibilidad y especificidad analítica para el ensayo ARCHITECT Syphilis TP para tres valores de corte S/CO: 1; 2,4 y 3,1, utilizando como método de referencia FTA-Abs y se obtuvo el índice de falsos positivos para el valor de corte declarado por el fabricante ${ }^{12}$.

\section{Resultados}

En la Tabla 2 se muestra la distribución de los resultados de la serología reactiva en el cribado. Entre los 246 sueros reactivos por ARCHITECT Syphilis TP se obtuvieron 129 sueros reactivos y 117 sueros no reactivos con V.D.R.L. (Figura 3). La consulta de la historia clínica de los pacientes indicó que, de los 129 sueros reactivos, 109 correspondieron a pacientes con diagnóstico clínico, dentro de los que se identificaron 39 casos de infección primaria, uno correspondió a una mujer gestante con un valor alto S/CO y V.D.R.L. de 1 dils y 20 correspondieron a pacientes en diferentes estadios de la enfermedad. Los 117 sueros de pacientes con serología discordante, es decir, reactivos en el cribado treponémico y no reactivos por V.D.R.L, fueron analizados mediante FTA-Abs, 20 sueros resultaron no reactivos (falsos positivos) y 97 sueros reactivos; de estos últimos, en 75 casos se comprobó que correspondieron a sífilis pasada, dos a infección primaria (Tabla 3 ) y los 20 casos restantes no

\begin{tabular}{|lc|}
\hline $\begin{array}{l}\text { Tabla 1. Características descriptivas de la población } \\
\text { estudiada con serología reactiva en el cribado } \\
\text { treponémico }(\mathbf{n}=\mathbf{2 4 6})\end{array}$ \\
\hline Variable & $\mathbf{n}(\%)$ \\
Sexo & $89(36,2)$ \\
Femenino & $157(63,8)$ \\
Masculino & $59(24,0)$ \\
VIH positivo & 7 \\
Femenino & 52 \\
Masculino & $10(4,1) *$ \\
\hline Mujeres gestantes & $15-84$ \\
\hline Edad (años) & 36 \\
Rango & \\
\hline Mediana & \\
\hline *Se incluye una mujer gestante positiva para VIH. \\
\hline
\end{tabular}

\begin{tabular}{|ccccc|}
\hline \multicolumn{4}{l}{ Tabla 2. Distribución de los resultados de serología para sífilis con el método } \\
quimioluminiscente ARCHITECT Syphilis TP y V.D.R.L \\
\hline ARCHITECT SyphilisTP & $\begin{array}{c}\text { V.D.R.L. } \\
\text { NR }\end{array}$ & $\begin{array}{c}\text { V.D.R.L. } \\
\text { R }(\mathbf{1}, \mathbf{2}, \mathbf{4} \text { dils) }\end{array}$ & $\begin{array}{c}\text { V.D.R.L. } \\
\text { R } \mathbf{2} \text { dils }\end{array}$ & $\begin{array}{c}\text { Total } \\
\text { (n) }\end{array}$ \\
R & 117 & 57 & 72 & 246 \\
\hline
\end{tabular}

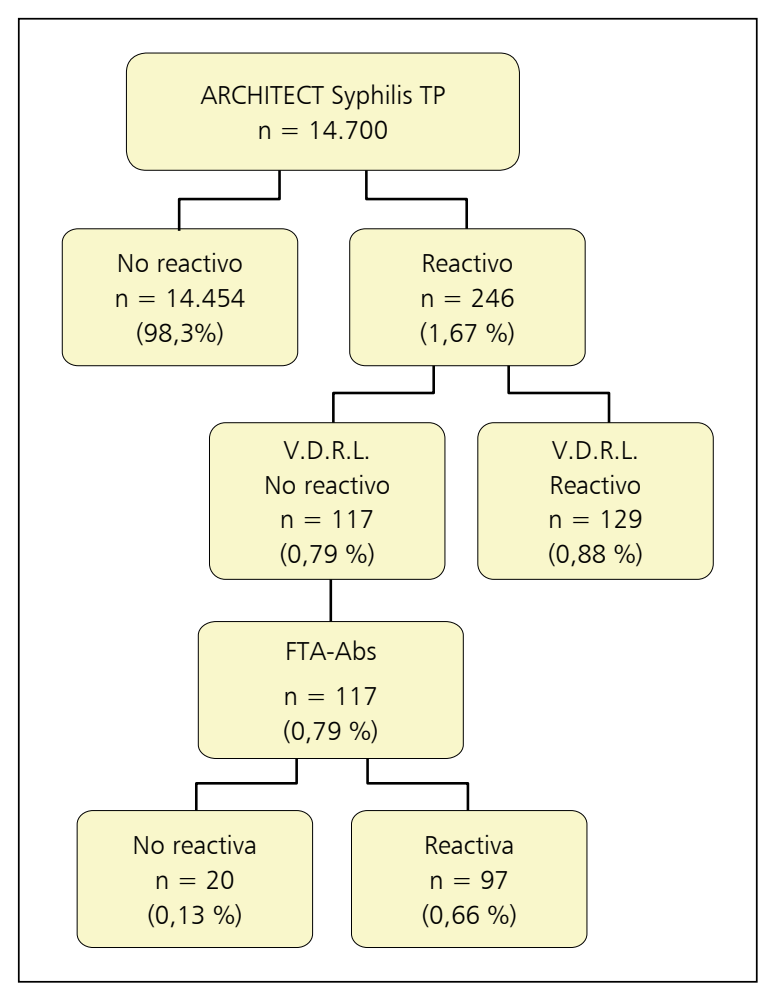

Figura 3. Resultados del cribado para sífilis utilizando el ensayo quimioluminiscente ARCHITECT Syphilis TP. 


\begin{tabular}{|c|c|c|c|}
\hline Paciente & $\begin{array}{l}\text { ARCHITECT Syphilis } \\
\text { TP (S/CO) }\end{array}$ & $\begin{array}{l}\text { V.D.R.L. } \\
\text { (dils) }\end{array}$ & FTA-Abs \\
\hline 1 & 13,56 & NR & $R$ \\
\hline 2 & 7,52 & NR & $R$ \\
\hline $2^{*}$ & 28,55 & 4 dils & $\mathrm{R}$ \\
\hline
\end{tabular}

FTA-Abs: absorción de anticuerpos treponémicos fluorescentes, NR: no reactivo, R: reactivo, V.D.R.L: Laboratorio de Investigación de Enfermedades Venéreas, 2* resultados obtenidos para el paciente 2 un mes después.

\begin{tabular}{|c|c|c|c|}
\hline $\mathrm{S} / \mathrm{CO}$ & $\begin{array}{c}1 \\
\begin{array}{c}\text { (Declarado por el } \\
\text { fabricante) }\end{array}\end{array}$ & 2,4 & $\begin{array}{c}3,1 \\
\left.\text { (Lee } y \text { cols }^{13}\right)\end{array}$ \\
\hline Sensibilidad (\%) & 100 & 100 & 98,1 \\
\hline Especificidad (\%) & 35,5 & 77,4 & 96,8 \\
\hline
\end{tabular}

pudieron ser corroborados por la historia clínica y/o no se solicitaron estudios posteriores para su evaluación. Entre los 20 resultados falsos positivos, se encontró que cuatro correspondieron a pacientes gestantes y tres a pacientes con infección por VIH. A su vez, estos 20 sueros que resultaron no reactivos por FTA-Abs tuvieron valores $\mathrm{S} / \mathrm{CO}$ comprendidos entre 1,01 y 3,45. En base a este hallazgo se calculó la sensibilidad y especificidad para distintos puntos de corte: $\mathrm{S} / \mathrm{CO}=1$, declarado por el fabricante del ensayo; $\mathrm{S} / \mathrm{CO}=2,4$ seleccionado de la población estudiada para una sensibilidad de $100 \%$ y una buena especificidad y $\mathrm{S} / \mathrm{CO}=3,1$ valor encontrado en el estudio de Lee y cols $^{13}$. Los valores de sensibilidad y especificidad analítica obtenidos al utilizar los distintos valores de corte se muestran en la Tabla 4. Para la población estudiada se obtuvo un índice de falsos positivos de $0,13 \%$.

\section{Discusión}

La implementación del algoritmo reverso para el cribado de sífilis constituyó un proceso gradual en el que se observaron ventajas o mejoras, tanto en utilidad diagnóstica como operativa; sin embargo, también se observaron algunas desventajas o dificultades en relación a costos e interpretación de los resultados. Entre las ventajas del cribado de sífilis con un método quimioluminiscente de alta sensibilidad, se destaca la detección de sífilis primaria recientemente adquirida en dos pacientes que presentaron el test reflejo V.D.R.L. no reactivo, que no podrían haberse diagnosticado tan tempranamente mediante el algoritmo tradicional ${ }^{14-16}$ (Tabla 3). La sensibilidad de los inmunoensayos quimioluminiscentes reportada por la bibliografía y el fabricante fue de $98-99 \%$ para sífilis primaria, coincidente con la encontrada en nuestra serie ${ }^{3,17}$. Mientras que la sensibilidad del método V.D.R.L. en la detección de sífilis primaria reportada por la bibliografía fue de $78 \%{ }^{3}$. Cabe destacar el resultado concluyente hallado para una mujer gestante con infección primaria que mostró una intensidad de señal alta $(\mathrm{S} / \mathrm{CO})$ en el inmunoensayo treponémico y resultado de 1 dils. con el ensayo no treponémico. Este resultado es especialmente relevante conociendo que el embarazo está considerado como una causa de reacción biológica falsamente positiva para V.D.R.L. ${ }^{18}$. Sin embargo, para esta paciente se obtuvo un resultado concluyente en el cribado. En relación con el aumento de la sensibilidad en la detección y considerando que la población de pacientes ambulatorios que concurre al laboratorio es de baja seroprevalencia para sífilis (menor a 2\%), se encontró un índice general de falsos positivos de $0,13 \%$, utilizando el valor de corte declarado por el fabricante S/CO: 1,0. El índice de falsos positivos ha sido evaluado por distintos autores; resultados similares a éstos se observaron en un estudio de Binnicker y cols. quienes usaron un EIA para el cribado mediante el algoritmo reverso en una población con baja prevalencia para sífilis ${ }^{19} \mathrm{y}$ encontraron un índice general de falsos positivos de $0,6 \%$, coincidente con el reportado previamente por el $\mathrm{CDC}^{9}$.

Se observó que, los resultados interpretados como falsos positivos $(n=20)$, tuvieron valores $S / C O$ comprendidos entre 1,01 y 3,45 . Lee y cols. ${ }^{13}$, mediante un análisis con curvas ROC utilizando el mismo inmunoensayo ARCHITECT Syphilis TP, informaron una sensibilidad de $82,7 \%$ y especificidad de $87,5 \%$, para el valor S/CO: 3,1 . Utilizando el valor de corte reportado por dichos autores, se encontró una sensibilidad y especificidad más elevadas (98,2 y 96,8\%, respectivamente), y en consecuencia, la disminución de resultados falsos positivos (Tabla 4). En base a estos resultados se consideró adecuado agregar un comentario interpretativo en el informe de los pacientes cuyos sueros presentaron señales $\mathrm{S} / \mathrm{CO}$ de 1,0 a 3,1 y con test reflejo no reactivo, sugiriendo al médico interpretar el resultado en el contexto clínico del paciente, evaluando la necesidad de confirmar mediante otra prueba treponémica o de repetir la serología en un lapso de 15 a 21 días para determinar seroconversión.

Se menciona que cuatro mujeres gestantes (de 10 analizadas) tuvieron resultados falsos positivos (CIA reactivo, V.D.R.L. y FTA-Abs no reactivos) y para estos casos se consideró adecuado incluir un comentario interpretativo en el informe y comunicar directamente con el médico solicitante. En la re-evaluación se obtuvo el mismo resul- 
tado inicial, sin observar seroconversión para V.D.R.L. y FTA-Abs. Es interesante destacar que una de las cuatro pacientes fue una mujer gestante con infección por VIH. Si bien es conocido que durante el embarazo se pueden obtener reacciones biológicas falsamente positivas con los métodos no treponémicos, los datos son limitados en relación a los ensayos treponémicos ${ }^{20}$. Mencionamos también tres pacientes con infección por VIH (de 59 analizados) cuyos resultados de serología fueron interpretados como falsos positivos (S/CO: 1,$3 ; 2,4$ y 3,7 ); uno de ellos se estudió 20 días después sin observar seroconversión para V.D.R.L. y FTA-Abs, mientras que en los otros dos no se realizó un seguimiento posterior o no se dispuso de datos serológicos previos. Por ello, no se pudo discernir si fueron verdaderos falsos positivos o pacientes con infección por VIH que recibieron tratamiento y en los que hubo sero-reversión de la prueba FTA-Abs, situación que se ha descrito en la bibliografía para este grupo de pacientes ${ }^{21}$.

Con respecto a las ventajas operativas, se hace hincapié en la automatización del cribado como recurso para reducir los tiempos de respuesta frente a la demanda creciente en el volumen de muestras analizadas por el laboratorio (700 pruebas mensuales en promedio), la mejora en la trazabilidad de las muestras, seguridad para el paciente, la simplificación del procedimiento manual laborioso, subjetividad en la lectura y eliminación del fenómeno de prozona atribuibles a la técnica de V.D.R.L.

Entre las desventajas, se destaca un aumento de 20 veces sólo en el costo de reactivo para el cribado mediante el algoritmo reverso respecto del tradicional, siendo el incremento más elevado si se incluye el costo adicional de las pruebas treponémicas confirmatorias necesarias para resolver resultados discordantes. Además, desde el punto de vista de costos en el sistema de salud, con este algoritmo, a diferencia del tradicional, se detectan pacientes que requieren tratamiento (sífilis primaria y latente) como pacientes que no requieren tratamiento (sífilis pasada y tratada o falsos positivos) y por esto, es fundamental una adecuada interpretación médica.

Una limitación de este estudio fue el uso del método FTA-Abs como segundo ensayo treponémico, debido a su menor sensibilidad para los estadios primario y latente tardío (78-96\% $)^{2,22}$, en lugar de la prueba TPPA recomendada por el CDC y las guías actuales del Ministerio de Salud de Argentina ${ }^{23}$. La FTA-Abs ha sido considerada el estándar de oro como método confirmatorio ${ }^{9}$, debido a su adecuada especificidad y en cada caso se realizó por personal del laboratorio entrenado y con experiencia en técnicas de inmunofluorescencia indirecta.
No pueden obtenerse conclusiones estadísticamente significativas sobre la eficacia del algoritmo reverso en la población de mujeres gestantes y de pacientes con infección por VIH, debido al escaso número incluido en esta serie.

Finalmente, conociendo que durante los últimos años se ha observado un incremento en la incidencia de sífilis en el mundo, y en especial en América Latina y el Caribe, tanto en población general como en la tasa de sífilis congénita ${ }^{24,25}$ y analizado las ventajas del algoritmo reverso con un método quimioluminiscente, se propone su implementación en laboratorios medianos a grandes que cuenten con la infraestructura adecuada y personal capacitado para asegurar la eficacia de este algoritmo y la correcta interpretación de los resultados en su población de pacientes.

Agradecimientos. A la Dirección del Laboratorio D’Agostino-Bruno por proporcionar la tecnología y los reactivos para llevar a cabo el estudio, especialmente a Elizabeth Masci y Patricia Lagoa, quienes se desempeñan como técnicas en el laboratorio.

\section{Resumen}

Introducción: La sífilis sigue siendo un problema de salud pública en todo el mundo; la precisión de las pruebas de diagnóstico es fundamental para el éxito de su control. Actualmente, hay dos enfoques para el diagnóstico serológico de la sífilis: el algoritmo tradicional y el algoritmo reverso. Objetivo: Analizar las ventajas y desventajas en la implementación del cribado para sífilis con el algoritmo reverso en un laboratorio clínico de pacientes ambulatorios. Materiales y Métodos: Se realizó un estudio de corte transversal analizando 246 sueros reactivos en el cribado sobre un total de 14.700 solicitudes de serología para sífilis. Se utilizaron los ensayos ARCHITECT SyphilisTP, V.D.R.L. y FTA-Abs. Resultados: De los 246 sueros reactivos por ARCHITECT Syphilis TP, 129 fueron reactivos y 117 no reactivos con V.D.R.L., éstos últimos resultaron 97 reactivos y 20 no reactivos por FTA-Abs, sugiriendo falsos positivos $(0,13 \%)$. Se detectaron dos casos de infección primaria, no detectados con V.D.R.L y un caso de infección primaria en una gestante con un valor alto S/CO y V.D.R.L. de 1 dils. Conclusiones: Entre las ventajas de utilizar el algoritmo reverso se encontró mayor sensibilidad en la detección de sífilis primaria; automatización, trazabilidad, interpretación objetiva y resultados concluyentes. 


\section{Referencias bibliográficas}

1.- Koneman E W, Allen S D, Janda W M. Diagnóstico Microbiológico, 5th ed. Editorial Médica Panamericana,1999, 927-36.

2.- Larsen S A, Steiner B M, Rudolph A H. Laboratory diagnosis and interpretation of test for syphilis. Clin. Microbiol. Rev. 1995; 8: 1-21. PMID: 7704889.

3.- Ratnam S. The laboratory of diagnosis of syphilis. Can J Infect Dis Med Microbiol 2005; 16 (1): 45-51. PMID: 18159528.

4.- Seña A C, Pillay A, Cox D L, Radolf J D. Treponema and Brachyspira, human hostassociated spirochetes. Jorgensen J, Pfaller M, eds. Manual of Clinical Microbiology, 11th ed. Washington DC: ASM Press, 2015, 1055-1081.

5.- Morshed M G, Singh AE. Recent trends in the serologic diagnosis of syphilis. Clin. Vaccine Immunol. 2015; 22: 137-47. doi: 10.1128/ CVI.00681-14.

6.- Seña A C, White B L, Sparling P F. Novel Treponema pallidum serologic tests: a paradigm shift in syphilis screening for the 21 st century. Clin. Infect. Dis. 2010; 51: 700-8. doi: 10.1086/655832.

7.- Organización Panamericana de la Salud. Prevención de la transmisión vertical de: sífilis, hepatitis B y VIH.2011. Disponible en http:// www.paho.org/arg/images/Gallery/SIDA/ guiaprevenviontv.pdf.

8.- Centers for Disease Control and Prevention. Syphilis testing algorithms using treponemal tests for initial screening-four laboratories, New York City, 2005-2006. CDC MMWR Morb. Mortal. Wkly. Rep. 2008; 57 (32): 872-5. PMID: 18701877.

9.- Centers for Disease Control and Prevention. Discordant results from reverse sequence syphilis screening-five laboratories, United States, 2006-2010. CDC MMWR Morb. Mortal. Wkly. Rep. 2011; 60 (5): 133-7. PMID: 21307823.
10.- Janier M, Hegyi V, Dupin N, Unemo M, Tiplica GS, Potočnik M, et al. European guidelines on the management of syphilis. J Eur Acad Dermatol Venereol 2014; 28(12): 1581-93. doi: $10.1111 / \mathrm{jdv} .12734$.

11.- Martínez Methol M S, De la Colina A, Masci E, et al. Concordancia entre un inmunoensayo quimioluminiscente treponémico y un test no treponémico en el screening para el diagnóstico de sífilis. VIII Congreso Argentino de la Calidad en el Laboratorio Clínico, Ciudad de Mar del Plata, 2014 (Póster I25)

12.- Clinical and Laboratory Standards Institute. User protocol for evaluation of qualitative test performance; Approved Guideline. 2nd ed.2008; documento EP12-A2.

13.- Lee K, Park H, Roh EY, Shin S, Park K U, Park M H, et al. Characterization of sera with discordant results from reverse sequence screening for syphilis. BioMed Res Intern 2013; Article ID 269347. http://dx.doi. org/10.1155/2013/269347.

14.- Tong M L, Lin L R, Liu L L, Zhang H L, Huang S J, Chen Y Y, et al. Analysis of 3 algorithms for syphilis screening serodiagnosis and implications for clinical management. Clin Infect Dis 2014; 58: 1116-24. doi: 10.1093/cid/ ciu087.

15.- Loeffelholz M J, Binnicker M J. It is time to use treponema specific antibody screening tests for diagnosis of syphilis. J. Clin. Microbiol 2012; 50 (1): 2. doi: 10.1128/JCM.06347-11.

16.- Marangoni A, Moroni A, Accardo S, Cevenini R. Laboratory diagnosis of syphilis with automated immunoassays. J. Clin. Lab. Anal 2009; 23: 1-6. doi: 10.1002/jcla.20268.

17.- Young H, Pryde J, Duncan L, Dave J. The architect syphilis assay for antibodies to Treponema pallidum: an automated screening assay with high sensitivity in primary syphilis. Sex Transm Infect 2009; 85: 19-23. doi: 10.1136/sti.2008.031872.

18.- Nandwani R, Evans DT. Are you sure it's syphilis? A review of false positive serology. international J STD \& AIDS, 1995; 6 (4): 2418. doi:10.1177/095646249500600404.

19.- Binnicker M J, Jespersen D J, Rollins L O. Direct comparison of the traditional and reverse syphilis screening algorithms in a population with a low prevalence of syphilis. J Clin. Microbiol 2012; 50: 148-50. PMID: 22090407.

20.- Mmeje O, Chow J M, Davidson L, Shieh J, Schapiro J M, Park I U. Discordant syphilis immunoassays in pregnancy: perinatal outcomes and implications for clinical management. Clin Infect Dis 2015; 61: 104953. doi: 10.1093/cid/civ445.

21.- Haas J S, Bolan G, Larsen S A, Clement M J, Bacchetti P, Moss A R. Sensitivity of treponemal tests for detecting prior treated syphilis during human immunodeficiency virus infection. J Infect Dis 1990; 162: 862-6. doi:10.1093/infdis/162.4.862.

22.- Park I U, Fakile Y F, Chow J M, Gustafson K J, Jost H, Schapiro J M, et al. Performance of treponemal tests for the diagnosis of syphilis. Clin Infect Dis 2019; 68(6): 913-8. doi: $10.1093 / \mathrm{cid} / \mathrm{ciy} 558$.

23.- Ministerio de Salud y desarrollo Social de Argentina. Diagnóstico y tratamiento de sífilis. Recomendaciones para los equipos de salud. 2019. Disponible en http://www.msal.gob. ar/images/stories/bes/graficos/0000001408c nt-2019-02-25_guia-sifilis.pdf.24 [consultado en abril de 2019].

24.- Organización Mundial de la Salud. Eliminación mundial de la sífilis congénita: Fundamentos y estrategia para la acción. 2008. Disponible en https:/www.who.int/reproductivehealth/ publications/rtis/9789241595858/es/ [consultado en junio de 2017].

25.- Ministerio de Salud y desarrollo Social de Argentina. Boletín sobre el VIH, SIDA e ITS en la Argentina, año XXI, Número 35, 2018 [consultado en abril de 2019]. $0000001385 \mathrm{cnt}-$ 2018-12-20_boletin-epidemiologico-vih-sidaits_n35.pdf 